\title{
Strengths and Weaknesses of the Young Black Men, Masculinities, and Mental Health (YBMen) Facebook Project
}

\author{
Daphne C. Watkins, Julie Ober Allen, Janelle R. Goodwill, and Blake Noel \\ University of Michigan
}

\begin{abstract}
The Young Black Men, Masculinities, and Mental Health (YBMen) project is a Facebook-based intervention that provides mental health education and social support to young Black men. The YBMen project was created to better understand and address the pressures and needs of young Black men, particularly with regard to issues related to their conceptualization of masculinity and mental health. Black men from a 2-year liberal arts college in the Midwest (United States) enrolled in the YBMen pilot project. The purpose of this study is to report what participants in the YBMen pilot project liked and disliked about the intervention, along with their suggestions for improvement. Qualitative results from the 8 Black men who actively participated in the YBMen Facebook intervention and completed the postintervention interview are reported. A systematic analysis identified 9 subthemes that described participants' reactions to different components and characteristics of the Facebook intervention. Results indicated that opportunities for relationship building and connectivity, coupled with engaging popular culture references used in the intervention encouraged young Black men to actively participate in the YBMen Facebook intervention. The YBMen project has potential to improve the health and well-being of young Black men by providing nontraditional resources that are easily accessible, culturally sensitive, and gender-specific. Implications of the YBMen project as an effective Internet-based program that promotes mental health and increases social support among young Black men are discussed.
\end{abstract}

Supplemental materials: http://dx.doi.org/10.1037/ort0000229.supp

$\mathbf{T}$ here is great promise in the use of Internet-based health education and social support programs focused on increasing self-efficacy for promoting and maintaining positive mental health (Watkins \& Jefferson, 2013). Online programs can provide skill building and role modeling, facilitate better health management, and enhance social support. The Young Black Men, Masculinities, and Mental Health (YBMen) project is a Facebookbased intervention that provides mental health education and social support for Black men ages 18 to 30 . Tailored to specific subgroups of young Black men, the YBMen project was developed to

This article was published Online First December 15, 2016.

Daphne C. Watkins, School of Social Work and Department of Psychiatry, University of Michigan; Julie Ober Allen, School of Pubic Health, University of Michigan; Janelle R. Goodwill, School of Social Work and Department of Psychology, University of Michigan; Blake Noel, School of Education, University of Michigan.

This project was supported by grants awarded to Daphne C. Watkins from the Vivian A. and James L. Curtis School of Social Work Research and Training Center and the Comprehensive Depression Center Phil Jenkins Award at the University of Michigan.

Correspondence concerning this article should be addressed to Daphne C. Watkins, School of Social Work, University of Michigan, 1080 South University Avenue, 60 Ann Arbor, MI 48109-1106. E-mail: daphnew@ umich.edu improve mental health, social support, and expand definitions of masculinity for young Black men using popular culture references that are familiar to them. This article reports the feedback received from young Black men who participated in the pilot YBMen project. Specifically, this article reports what the participants liked and disliked about the YBMen Facebook intervention, as well as their suggestions for ways to improve the intervention in future iterations of the project.

For more than two decades, mental health professionals have been concerned about the disproportionate increase in suicide among adolescent and young adult Black males (Joe, Baser, Breeden, Neighbors, \& Jackson, 2006). The largest epidemiologic study of Blacks in the United States found that Black men aged 34 and younger experienced psychological distress at higher rates than did those over the age of 35 (Lincoln et al., 2011; Sellers et al., 2009). This suggests that psychological distress among young Black men is a problem requiring special attention. Due to the stigma associated with psychological distress, it persists as a "silent" problem in Black communities, and the tendency to suffer in silence makes this condition pernicious throughout African American communities. Though communities witness the direct and indirect effects of distressed Black men, it is difficult to gauge if the problems these men experience should be addressed via formal mental health interventions or informal community-based interventions. 
Early adulthood represents a critical opportunity for early intervention to support the mental health and well-being of young Black men. Early interventions can improve young Black men's present and future mental health and well-being by imparting knowledge, enhancing skills, and introducing supportive resources, all of which may continue to benefit men over the life course. Mental health promotion programs for young Black men can support the establishment of lifelong, healthy coping habits and provide alternatives to the unhealthy coping behaviors often accessible in Black communities, such as unhealthy eating, alcohol consumption, drug use, and a sedentary lifestyle (Jackson, Knight, \& Rafferty, 2010; Mezuk et al., 2013). Additionally, mental health promotion for young Black men may prevent negative, cumulative effects of chronic stress on the body, thus preventing the development of chronic diseases later in life, as this has been posited as a key predictor of racial health disparities (Braveman, Egerter, \& Williams, 2011).

Social support is a reciprocal process involving social networks of close relationships (significant others, family, and friends) and weak ties (coworkers, acquaintances, and strangers) (Wester et al., 2006). Social support influences health by regulating thoughts, feelings, and behaviors to promote health; fostering an individual's sense of meaning in life; and facilitating health-promoting behaviors (Paxton, Robinson, Shah, \& Schoeny, 2004). Research suggests that individuals who obtain higher levels of social support report better health status, psychosocial adjustment, coping behaviors, health promotion behaviors, quality of life, well-being, and self-actualization (Paxton et al., 2004). Similarly, the capacity to establish and sustain friendships is a critical indicator of healthy psychological development and friend support has been linked to outcomes such as improved physical and mental health, coping efficacy, and self-esteem.

With the popularity of social media, social support can now be provided face-to-face, over the telephone, and via the Internet (i.e., online). Online social support groups are often considered virtual communities and their use has quadrupled over the last decade (Ellison, Vitak, Gray, \& Lampe, 2014; Oh, Ozkaya, \& LaRose, 2014). Various tools are used in online social support groups such as web-based message boards, newsgroups, videoconference meetings, listservs, chat sessions, e-mail exchanges, and discussion forums (Englar-Carson \& Kiselica, 2013). Typically, self-help in nature, rather than therapeutic, online support groups share similar goals as face-to-face support groups; however, online groups provide a certain amount of anonymity that one may not experience in a face-to-face support group setting. Anonymity facilitates the discussion of potentially embarrassing topics or otherwise taboo subjects, increases the potential of self-disclosure, and encourages honesty and intimacy among the participants when discussing potentially stigmatizing topics, such as those related to mental health (Oliver, 2006; Payne, 2011; Watkins \& Jefferson, 2013). Compared with face-to-face groups, online support groups offer greater access and are more convenient, but they are less spontaneous than face-to-face encounters as they rely heavily on written text and nonverbal responses (Watkins \& Neighbors, 2007; Payne, 2012). Mixed evidence suggests that social support can increase among young adults via online engagement and generate positive mental health outcomes typically associated with social support. For example, Oh and colleagues (2014) found that frequent e-mail exchanges increased perceived social support, which alleviated depression among their college sample. On the contrary, depressive symptoms have been found to increase when participants engaged in e-mail and instant-messaging use (Wang, Wu, \& Liu, 2003).

Social support among Black males is grossly understudied, yet research suggests that men who engage in higher levels of social support have better health outcomes, role functioning, psychosocial adjustment, coping behaviors, quality of life, well-being, and self-actualization (Wang et al., 2003). A supportive online community may provide young Black men with a convenient, nonthreatening space (Pinnock, Jones, \& the Education Committee of the Australian Prostate Cancer Collaboration, 2003) that is accessible 24 hours a day. This space can be used to discuss topics that make face-to-face groups of young Black men uncomfortable, such as stress, racism, sexism, or varying conceptualizations of masculinity (Chang \& Yeh, 2003; Mulveen \& Hepworth, 2006). Furthermore, it has been found that men of all races who participate in online social support groups are more empathetic to the problems experienced by other men, which improve their longterm relationships (Chang \& Yeh, 2003; Pinnock et al., 2003). This empathetic response may not always be shared, or welcomed, during face-to-face all-male support group settings due to the stigma associated with being an emotive man (Pinnock et al., 2003).

\section{What is the YBMen Project?}

The YBMen project is a 5-week mental health education and social support intervention for young Black men that was pilot tested in 2014. The YBMen project was created to better understand and address the unique pressures and needs of young Black men, especially regarding issues related to masculinity and mental health. For the YBMen pilot project participants were recruited, screened, and then divided into two groups: the intervention group (i.e., which participated in the YBMen Facebook intervention) and the comparison group (i.e., which did not participate in the YBMen Facebook intervention but were provided with an educational newsletter via e-mail on masculinity, social support, and mental health at the conclusion of the study).

Intervention group members joined a private Facebook group created and monitored by the YBMen study team. Over the course of the project period, the study team posted gender- and culturally relevant prompts from popular culture references (e.g., song lyrics, images, YouTube videos, current headlines) to educate YBMen Facebook participants about the importance of mental health, social support, and the challenges associated with a rigid adherence to masculine norms. The study team posted daily prompts and questions designed to generate group discussion and facilitate the development of a supportive online group in which men communicated with each other via comments, new posts, "likes," and "shares." Group facilitation techniques related to action planning and feedback, group problem solving, and individual decision making to improve mental health behaviors and outcomes were used by the team to engage participants in learning, skill development, and creating a supportive atmosphere for examining each week's topic. The YBMen pilot Facebook topics are in Online Appendix A. 


\section{The YBMen Project Conceptual Framework}

The conceptual framework for the YBMen project is derived from the integration of evidence on the importance of addressing social determinants of health, the appropriateness of incorporating culture and gender in interventions to improve health among young Black men, and the theory of online social support. First, because previous research has described the influence of social determinants on physical and mental health (Griffith, Gunter, \& Watkins, 2012; Chae, Lincoln, \& Jackson, 2011; Watkins \& Griffith, 2013; Watkins, Hudson, Caldwell, Siefert, \& Jackson, 2011), we focused on understanding young Black men in the context of key social determinants affecting the lives and well-being of this group (e.g., socioeconomic position, race, masculine identities). Second, research suggests behavioral interventions that target young Black men need a culturally appropriate and gender-specific approach that addresses their unique characteristics and experiences (Watkins, 2012; Rowling, 2006). The impetus for using these approaches has origins in community-based participatory research as well as research on interventions that are empowered by and for young Black men. Finally, we used the theory of online social support (OSS; LaCoursiere, 2001) to address gaps in previous models aimed at understanding and improving young Black men's health in the context of their social environments. The purpose of OSS theory is to describe health information-seeking behaviors that occur on the Internet through support from interpersonal relationships with other unseen persons (LaCoursiere, 2001). The YBMen project is guided by a conceptual framework derived from the integration of the theory of OSS, social determinants of health, and the role of culture and gender in health interventions.

\section{Rationale for the Current Study}

Despite growing interest in the use of online tools for promoting health and well-being, few studies have focused primarily on young Black men. Of the health studies that have used Internetbased tools with Black men, many have focused on either gay or bisexual men (Chang \& Yeh, 2003) or men with prostate cancer (Pinnock et al., 2003). More efforts should be put toward developing innovative interventions that translate current research findings on young Black men into practical solutions that can effectively recruit, retain, and improve health outcomes for young Black men (Watkins \& Griffith, 2013; Watkins \& Jefferson, 2013). Before the scalability of such efforts can be considered, interventions must first be developed and tested for their culturally sensitive and gender-specific feasibility and attractiveness among groups of young Black men. There is a need for low-cost, highimpact interventions for young Black men that use a medium that is a part of their daily lives: social media. Furthermore, the ability to meet young Black men where they are by using popular culture references to educate them about mental health, their definitions of manhood, and social support is an untapped opportunity, particularly because the three are so strongly associated (Watkins, 2012; Watkins \& Griffith, 2013; Watkins \& Jefferson, 2013). The purpose of this study is to report the qualitative findings from the pilot YBMen project. Specifically, we were interested in learning what participants liked and disliked about the YBMen project Facebook intervention and what their suggestions were for ways to improve the YBMen project Facebook intervention in the future. Knowing what young Black men like and dislike about the YBMen Facebook intervention is useful to researchers and practitioners who think about ways to maximize interventions for this subgroup that are delivered through social media platforms, such as Facebook.

\section{Method}

\section{Study Design}

The original YBMen pilot project used a quasi-experimental, pretest-posttest mixed-methods design (Watkins et al., 2015) that occurred over 5 weeks with 30 young Black men. Data collection for the pilot consisted of a closed-ended survey and an in-depth interview that occurred at pretest (Time 1), immediately postintervention (Time 2), and 8 weeks after the conclusion of the intervention (Time 3). Though the original YBMen pilot project included both quantitative and qualitative measures, the present study is exploratory by nature and reports only findings from the in-depth interviews administered to the YBMen Facebook intervention group postintervention. Specifically, data about the YBMen Facebook group likes, dislikes, and suggestions are underscored in the current study because this information addresses the research questions posed by the study team that pertain to the overall effectiveness and feasibility of the YBMen Facebook intervention. The institutional review board at the lead author's institution reviewed all study protocols and materials prior to implementation.

\section{Sample}

The original YBMen pilot included participants who identified as Black men aged 18 to 26 , who were enrolled in the college that served as the pilot study site, and who had never been diagnosed with a mental health condition by a medical professional. Men previously diagnosed with a mental health condition were excluded because the YBMen Facebook intervention was designed to address subclinical distress and depression symptoms, provide education, and focus on prevention, not treatment; addressing more serious mental health disorders were beyond the goals of the intervention given the complexity and diversity of mental health disorders and treatment modalities. Because of the exploratory nature of the original YBMen pilot, it was determined that project resources would support developing and testing the pilot phase of the YBMen project with 30 young Black men. Therefore, the original pilot project involved collecting primary qualitative and quantitative data from all 30 participants and implementing a 5-week intervention via Facebook with half of the participants. Thirty Black men from a 2-year liberal arts college in the Midwest (United States) were enrolled in the YBMen pilot; however, 11 were assigned to the intervention group. Of these 11 men in the intervention group, 8 completed the in-depth interview about what they liked and disliked about the YBMen Facebook intervention and provided suggestions for ways to improve it. The current study reports on the eight in-depth interviews with the YBMen Facebook group participants who reported on the YBMen Facebook intervention at Time 2 (postintervention). 


\section{Data Collection}

The YBMen project director (lead author) is a Black/African American female researcher with over 13 years of experience doing research and community interventions on the mental health of Black men. Other study team members were Black and White graduate students (male and female), many of whom had experience working on previous research projects related to racial and ethnic health disparities. Study team members assisted with recruiting and screening the pilot YBMen project participants, collecting surveys, and interviewing participants. The staff at the study site's multicultural center and Black men who had already enrolled in the study (i.e., snowball sampling) also assisted with recruitment efforts.

Qualitative procedures included in-depth interviews with YBMen Facebook intervention group participants at Times 1, 2, and 3. The qualitative, in-depth interview questions addressed (1) the YBMen Facebook intervention (e.g., "What did you think about the things that were posted in the YBMen Facebook group by our project staff?"), (2) mental health (e.g., "Do you think Black men's experiences with mental health are different than that of men from other racial groups?"), and (3) masculinity (e.g., "Do you think Black men's experiences with masculinity are different than that of men from other racial groups?"; see Online Appendix B for the full list of questions). Interview questions underscored in the current study help to assess what characteristics of the YBMen Facebook intervention participants liked, what characteristics they disliked, and what suggestions they had for ways to improve the YBMen Facebook intervention in the future.

For the purpose of this study, we report only the findings from the Time 2 (postintervention) in-depth interviews with the YBMen Facebook intervention group participants. Eight $(n=8)$ of the 11 intervention group participants participated in the YBMen Facebook group and completed Time 2 in-depth interviews. We did not intentionally exclude the 3 less-active YBMen Facebook group participants from doing a postintervention interview with us; but rather, they where not present for the postintervention interviews. Study members matched by race and gender (i.e., Black men) conducted most of the in-depth interviews. Interviews lasted between 30 and 90 minutes each. Interviews occurred in classrooms at the study site at times that were mutually convenient for the YBMen Facebook participants and members of the study team. Participants were offered a cash incentive $(\$ 15)$ for their completion of the Time 2 (postintervention) interview.

\section{Data Analyses}

A team approach guided the qualitative analysis for this study (Watkins, 2012; Watkins \& Gioia, 2015). First, the analysis team completed preparatory steps (e.g., transcribing notes and audiorecordings verbatim, formatting transcripts, and reading through the transcripts) required for team-based qualitative data analyses (Fernald \& Duclos, 2005; Watkins, 2012). A spreadsheet technique was used to organize, manage, and analyze the data). First, an "all-inclusive data table" was developed using Microsoft Word. The all-inclusive table included seven column headings: transcript number, questionnaire section, question asked, participant's response, notes, code, and theme. After the data table was created, the analysis team applied a data reduction technique developed by the lead author called the "rigorous and accelerated data reduction" (RADaR) technique (Watkins \& Gioia, 2015; Watkins, Wharton, Mitchell, Matusko, \& Kales, 2015). The purpose of the RADaR technique is to reduce (i.e., condense) qualitative data in a way that generates results quickly and rigorously for project deliverables such as manuscripts, final reports, and/or health promotion materials (Watkins \& Gioia, 2015).

For this study, data reduction tables underwent four reduction phases, and each represented a more narrow and specific presentation of the qualitative data. To reduce the qualitative data, the analysis team reviewed the all-inclusive data table and made notes about areas of commonality and overlap between YBMen participants. The team then generated opinions about the relevance of certain quotes and the intersections of concepts. As a part of the data reduction process, coding procedures were used that allowed segments of raw text to be identified and compared with other segments and analyzed for embedded meaning. A two-level coding process was used. The first level of coding (i.e., open coding) identified preliminary text segments that were used to identify categories, concepts, and themes germane to the overall study goals. This first level of themes (e.g., likes, dislikes, and suggestions) required further in-depth exploration, so the team worked individually, then collectively, to reduce and code the data. A more "focused" level of coding (Grinnell \& Unrau, 2014; Watkins \& Gioia, 2015) was used to examine subthemes and this second level of coding and analysis captured a deeper understanding of the likes, dislikes, and suggestions expressed by the YBMen project Facebook participants.

\section{Results}

\section{Demographics}

Of the 30 young Black men enrolled in the original YBMen pilot project, 11 were placed in the intervention group. Of these 11, 8 participated in the YBMen Facebook group and completed the in-depth postintervention interviews where we collected data about the participants' likes, dislikes, and suggestions. Characteristics of the YBMen Facebook intervention group members are described in Table 1. Baseline depressive symptom scores indicated that some intervention participants (between 26\% and 66\%) met criteria for depressive symptoms, though none reported severe impairment associated with their symptoms (Watkins et al., 2015). Sixtyseven percent of the YBMen Facebook group members viewed postings at least once a week during the 5-week intervention and approximately $51 \%$ actively participated by posting comments and materials in the group.

\section{YBMen Project Participant Perceptions}

Our analysis focused primarily on the following research questions: What characteristics of the YBMen Facebook intervention did participants like? What characteristics did they dislike? What suggestions did they have for ways to improve the YBMen Facebook intervention? Therefore, likes, dislikes, and suggestions served as the primary themes for this analysis (Online Appendix C). YBMen Facebook intervention participants reported five characteristics about the project that they liked and one characteristic 
Table 1. Characteristics of the Young Black Men, Masculinities, and Mental Health (YBMen) Pilot Intervention Group $(n=11)$

\begin{tabular}{lc}
\hline \multicolumn{1}{c}{ Demographic characteristic } & Percentage \\
\hline African American men & 100 \\
Average age (years): 19.9 & \\
Age range: $18-24$ & 45.5 \\
Employed, in addition to attending college & 9.1 \\
Married or had a significant other & \\
Participants who were at risk for depression ${ }^{\text {a }}$ & \\
Time 1-Baseline scores $(n=11)$ & 65.6 \\
$\quad$ Determined by the PHQ-9 & 26.3 \\
$\quad$ Determined by the GMDS & 37.5 \\
Time 2-Postintervention scores $(n=8)$ & 25.0 \\
$\quad$ Determined by the PHQ-9 & \\
$\quad$ Determined by the GMDS & 67.3 \\
Participation in the YBMen Facebook intervention $(n=11)$ & 50.9 \\
Viewed Facebook postings weekly & \\
Posted comments or new material on Facebook each week & \\
\hline
\end{tabular}

Note. PHQ-9 = Patient Health Questionnaire; GMDS = Gotland Male Depression Scale.

${ }^{\text {a }}$ Lower depressive symptoms were determined by changes in scores.

that they did not like. They also shared three suggestions for ways to improve the project in future iterations. We report on each of these in the subsequent section.

\section{YBMen Project Likes}

Our analysis identified five subthemes under the "likes" theme. Specifically, YBMen participants noted that they liked the intervention because it (a) was educational and thought provoking, (b) allowed them to build relationships and support, (c) had good content, (d) used Facebook as the delivery platform, and (e) produced positive participant outcomes.

Educational and thought provoking. There were aspects of the YBMen project that participants liked overall, such as its ability to bring Black men together to discuss topics that are germane to them. Participants agreed that the YBMen project was educational; as one noted,

[The YBMen project] was a great thing . . . something I . . . haven't done in my life before, but I like it. It's educational and you can learn a lot from it, like we're teaching y'all and y'all teaching us in a way. Like both . . . it's a learning environment. That's what I say.

Participants also found the group to be thought provoking. For example, one participant noted how topics in the group were things that he ruminated over, even days after they were posted:

I think [the YBMen Facebook posts] were thought provoking. I think they were worded in such a way that it made you really think about it ... some stuff you could think about it right then and there. But other things, I would take a few hours to think about them.

During postintervention interviews, the participants also discussed their understanding of masculinity and manhood before their participation in the group compared to how they felt about these topics after the intervention ended. In particular, participants enjoyed being challenged to think about notions of masculinity
("Before this, I never really thought about masculinity, but [participating in the YBMen project has] made me think about it more, and . . . I'll continue to think about it more.") Similar reactions were reported for mental health, as participants also commented on the increased depth of their understanding of mental health during the YBMen project:

[The YBMen Facebook group] made me think more in depth ... . about mental health and what the causes are. It made me look into research about mental health more than I would. I would have never thought about mental health before you guys came.

Study participants also reported that being connected to other young Black men through the intervention served as a critical component in shaping their overall thought processes and experiences during the project.

Building relationships and support. The opportunity to be part of a group where they felt supported and could convene with other young Black men was important to participants. The YBMen project built new relationships, and strengthened old ones. For one participant, the chance to build relationships with other men was what attracted him to the group. He stated,

\begin{abstract}
One thing that made me really want to participate [in the YBMen Facebook group] was just seeing other people's comments and ... seeing the other challenges that they went through that I went through, too. I even commented on [the post by] a friend of mine that was going through something that I didn't even know [about]. I told him ... how I overcame it. . . . I felt inspiring, to be honest; it was just enlightening.
\end{abstract}

In a world where young Black men often feel like they are under a microscope, many YBMen Facebook group participants liked being able to express their opinions freely in the YBMen group with other Black men, as they rarely had an opportunity to do this in their daily lives. Many participants felt that the YBMen group was a welcoming and nonjudgmental space where they could discuss difficult topics with other Black men. One participant noted,

I would say I really enjoyed being in a place where I could hear the opinions of other Black men ... No one's trying to impress anyone. . . Everyone's just being themselves and giving their opinions. ... Every now and then I [want to] talk about some really deep topics and I feel like with the YBMen group it's 100\% that. ... That was really nice "cause I do not get that often, not with Black men.

The fact that participants liked building relationships in the YBMen group underscored the importance of connectedness in providing a place of refuge where they could safely disclose and reflect upon issues that were important to them. This finding accentuated participants' comfort with discussing topics that may be taboo when discussed in other social spaces.

YBMen intervention content. Participants reported that they liked the YBMen Facebook intervention content. They discussed the questions and prompts posted in the YBMen Facebook group that were used to generate discussion between participants For example, one participant stated, "I think [the YBMen Facebook intervention posts were] pretty good. It was to the point and it was straight forward . . real. I liked the questions, you know." Rather than focus solely on the questions that were posed during 
the YBMen Facebook group, another participant discussed the intervention topics in general,

Just about every topic [in the YBMen Facebook group] was engaging. Honestly . . . all of them were good posts and good approaches. If anything, they just made me curious you know? . . . it depends on how you want to look at it, but I think it was good stuff.

The study team was intentional in their efforts to establish content that was culturally relevant to this population by using a variety of mediums (i.e., YouTube videos, news articles, etc.), to increase the likelihood of the YBMen Facebook content being applicable to and well-received by the participants. In doing so, study participants made links between content posted by group administrators and their individual interests and experiences, which they described during the interviews.

Facebook as the intervention platform. Use of the social media platform, Facebook, was also an aspect of the project that the participants said they liked. Many noted how they were always logged into Facebook on one or multiple electronic devices: "I'm always around my Facebook. I have it on my phone, at my house, on my laptop ... a at school." When asked about using Facebook to deliver the YBMen intervention, other participants underscored its popularity with young adults, ". . . lots of teens are using it nowadays and young adults. And it's an easier way. . . . For me, it got sent to my phone, so that was an easy way for me to view it. . .." Yet, one of the more frequent comments about the benefits of using Facebook as the platform for delivering the YBMen project was the privacy it provided for the YBMen participants. "[YBMen's use of Facebook] was a good way to express yourself, most people really can't do it like just having a conversation with someone so with this, they will go onto the Internet." When reflecting on concerns surrounding feasibility and access to culturally sensitive mental health promotion resources for young Black men, Facebook seemed to be an effective way to disseminate information to them.

Produced positive participant outcomes. Another subtheme under the YBMen projects "likes" theme was the immediate benefits of participating in the YBMen project (e.g., one participant told our interviewer a story about some recent traumas in his life). He shared,

I had an incident about two months ago where a friend of mine was murdered ... It was nice going on to the YBMen group and seeing that all these other Black men who all come from different walks of life ... we're still striving to be better and do better things. It was a motivational tool . . . I actually stopped drinking half way through [the YBMen project] entirely. I do not drink [anymore] and that's from like two or three times a week ... After I got back to school Monday [following a drinking binge following a friend's candlelight vigil], I checked the YBMen group and I was just reading a couple posts and I was like, you know what, I just need to do more positive things with my time.

Other participants shared stories about how they were able to use the YBMen Facebook group to cope with some of the challenges they were experiencing; for example, one participant noted, "The YBMen project] gave me something to do to take my mind off [my personal struggles]. The stuff I was saying [in the YBMen Facebook group], I have never told anybody." The connectivity among participants emerged as a reoccurring pattern that led to positive experiences within the group and greater overall participant satisfaction. Despite this however, one part of the intervention posed a challenge, which alerted the research team to explore what was disliked by participants.

\section{YBMen Project Dislikes}

Our team identified one subtheme under the "dislikes" theme, and it involved the type of language used in the YBMen Facebook group. For example, one participant noted "I didn't understand some [words] that were used . . . the stuff that I posted is the stuff that I understood. And there was some stuff that I didn't understand, so I just kind of avoided it." Unfortunately, not understanding a word or the language used by the YBMen Facebook group moderator sometimes meant that group members did not participate in that discussion topic. "The stuff that I really didn't understand, I just didn't comment. Usually it was 'cause of the wording or I just didn't understand ... what you wanted from that question." Language issues in the intervention highlighted the importance of cultural sensitivity and specificity and recognizing that the language and jargon used by subgroups of young Black men may differ based on geography and other characteristics; these characteristics can play an important role in effective health communication.

\section{Suggestions for Improving the YBMen Project}

In an effort to improve future iterations of the YBMen project, our team was especially interested in subthemes identified under the theme "suggestions." YBMen Facebook group participants suggested that we include (a) better wording/language, (b) more media and popular culture references, and (c) face-to-face meetings.

Better wording/language. During the interviews, the wording and language used in the YBMen Facebook group arose as a frequent topic of discussion, as indicated previously. The men suggested that more relevant language and familiar wording be used to discuss topics in the group. Similarly, though the YBMen Facebook group administrators consulted with young Black men about the language used in the Facebook intervention during project conception and early planning, it became clear that some words (despite their popularity with some groups of Black men) were not familiar to all groups of Black men. An example of this was the Facebook administrator's use of the word grit, which means persistence and resilience to Black men of certain ages and geographic locations in the United States, but was unfamiliar to the men in our study:

... [The word grit] ... I never heard this used like this before ... it did help us expand our vocabulary. But at the same time, it made people not answer 'cause they do not know what we was talking about and most of them may be too lazy to look it up. . . Basically when you saying certain things people do not know, we tend not to answer.

This emphasizes the heterogeneity among young Black men and the importance of carefully identifying appropriate language for a specific group, while simultaneously debunking the antiquated stereotypes that young Black men are a monolithic group.

Expansion of popular culture references and media. Another topic that came up during the in-depth interviews was the desire for more media and popular cultural refer- 
ences. For example, participants wanted the YBMen Facebook group administrators to post more song lyrics and quotes from familiar music artists as a way to provoke discussions on the Facebook wall: "Use rappers' quotes. I think it will most likely touch us "cause we know what they [are] saying, we [have] listened to it before." Though lyrics were a popular suggestion, so was the inclusion of more topics germane to college-aged Black men. Another participant suggested that we post topics and images young Black men are likely to encounter in their daily lives, "Yeah, things that we see every day. We college kids see sex, drugs . . . violence. . . . Especially from where I'm from, I still see [bad things] every day." The use of more multimedia, especially expanding the use of videos, was another suggestion. Largely, participants wanted to see more innovative ways to introduce topics and spark discussions in the Facebook group, beyond the use of text. One stated the following:

I would have liked to see more videos though, to be honest. A little more videos about what ya'll [are] taking about, you know? Like masculinity, and let me think about it a little bit . . I'll be bound to click on it to see what type of message it was.

These specific requests for increased usage of videos, songs, and other media outlets suggests that popular culture not only plays a potentially significant role in shaping young Black men's perceptions and conceptualizations of masculinity, manhood, and mental health, but also can be harnessed to promote engagement and encourage reflection on these issues.

Have face-to-face meetings. Participants proposed that the YBMen project team implement more in-person meetings with the study participants. Though the research team thought that keeping the intervention solely Internet-based would be a strength of the project, some study participants commented on the value of occasional face-to-face meetings. One participant requested " . . . if possible [we could] meet once a week, every week . . . just like . . . have a group," and he was not the only participant who thought more face-to-face interaction would be a benefit to the YBMen intervention. For example, another participant stated that “. . . if it was manageable, having a meeting between the beginning and the end [of the intervention], like right smack in the middle, seems like an interesting idea to me." Our team implemented the YBMen project with an established men's group on campus, which likely influenced the men's participation as well as their trust for the research team. Not only did participants like that we did this, but they suggested that we work with other established groups of Black men for future YBMen projects.

I think [running YBMen through our established group on campus] does play a role ... and I think that was a really good move on you guys. I was happy just to be part of the project but already having an established group that works together... it helps.

\section{Discussion}

The purpose of this study was to report the YBMen pilot project participants' likes, dislikes, and suggestions for improving the YBMen Facebook intervention. This information was important to our team because the overall YBMen project outcomes included reductions in depressive symptoms and increased social support among participants (Watkins et al., 2015). These data will inform future iterations of the YBMen Facebook intervention as we continue to improve, refine, and further target the intervention for different groups of young Black men. The interviews also created mutually beneficial exchanges between our research team and the young Black men enrolled in the study. Our focus on the "likes" allowed us to gather participants' opinions about successful components of the intervention that we can retain and enhance. For example, a key feature of the YBMen Facebook intervention was that it gave participants the opportunity to build relationships and support with other Black male peers, which has been noted as beneficial in previous studies with young Black men (Adams \& Turner, 2013; Harper, 2013; Jackson, 2012; Scott et al., 2014).

In addition to gaining insight into what features of the YBMen Facebook intervention participants liked, this study provides insight about what they did not like. The main aspect of the intervention that participants were critical about was some of the word choices used in the YBMen Facebook intervention. Other studies have discussed the use of language when engaged in research and practice with African Americans overall (Gitlin, Harris, McCoy, Hess, \& Hauck, 2015) and African American men specifically (Odedina et al., 2014; Watkins \& Neighbors, 2007). This is an area that our study team will need to explore and improve for future YBMen Facebook interventions. It is also an area that should be taken into consideration by researchers and practitioners who work directly with young Black men. Also, although participants enjoyed the references to popular culture (e.g., YouTube videos, images, songs, current headlines, athletes, music artists, and actors), they wanted more. Previous studies have also noted the value of media messages in educating African Americans and combating stereotypes (Holt, 2013; Verney, 2013).

Young Black men are not a monolithic group. Although there are certain experiences associated with being a young Black man in America that may resonate across groups, not everything relevant to one group of young Black men will translate to another group of Black men in the same age group. This is why previous studies have underscored the importance of tailored and targeted efforts focused on Black men (Griffith, Allen, Johnson-Lawrence, \& Langford, 2014; Griffith, Metzl, \& Gunter, 2011; Watkins, 2012; Watkins \& Griffith, 2013; Watkins \& Jefferson, 2013), so that their unique experiences can be included in efforts to improve their mental health and well-being. It is also important for interventions aimed at young Black men to be cognizant of the vernacular and lexicon used within specific geographical regions, because language is a fluid form of communication that can serve as either a barrier or a facilitator to mental health care and educational resources for young Black men.

We believe that the careful attention given to the unique experiences of young Black men in the YBMen Facebook intervention is what resulted in such favorable responses by the participants. Although some researchers may be impartial to comments such as "Before this, I never really thought about masculinity, but [participating in the YBMen project has] made me think about it more," the statement that directly followed this comment (“. . . and I'm pretty sure I'll even continue to think about it more ...") confirms that we are on the right track with regard to educating young Black men about the importance of making connections between their mental health, definitions of masculinity, and social support. The original purpose of the YBMen project was to educate Black men about issues involving their mental health, masculinity, and social 
support. Young Black men in our study reported that the YBMen Facebook intervention helped them think more about masculinity and their own definitions of manhood. This finding has implications for the inclusion of masculinity and manhood in new and existing health and well-being interventions for Black men. In many ways, our findings echo that of previous research from Black psychology (Belgrave \& Allison, 2010; Parham, White, \& Ajamu, 1999), which has emphasized the importance of African-centered approaches to treatment. Though the YBMen project does not address severe mental disorders, we align our thinking with those of previous scholars who acknowledged the importance of culture and adaptation in addressing matters that involve the mental health and well-being of Black men.

Although providing a more anonymous space, via online interactions, was an intentional approach of the YBMen project, given previous research on the low use of more traditional mental health support services among young Black men and the stigma associated with mental health issues among this group (Regehr et al., 2013), some of our participants indicated that they would like more face-to-face interactions with us and other participants from the group. The pilot project was associated with an existing men's group, and even though not all our participants were members of the men's group, this context may have influenced participants' desires for more in-person contact. Further research is needed to determine whether adding an in-person component to the current social media delivery and engagement format would enhance the effectiveness of the YBMen Facebook intervention.

Aside from battling concerns that are typically associated with seeking mental health care amid the threat of stigma, access remains a looming threat for young Black men that may be interested in receiving resources or information related to this topic (Thorpe et al., 2016; Woodward, Taylor, \& Chatters, 2011). Visiting a therapist is both time-consuming and costly, which in turn prevents many people from underserved communities from utilizing these services. There is also no guarantee that the mental health provider will be sensitive to the unique cultural experiences of young Black men. Therefore, social media is a currently untapped resource in mental health promotion within this specific group (Watkins \& Jefferson, 2013). The results of the YBMen pilot project suggests that the implementation of a sustainable online intervention could be effective in alleviating disparate mental health occurrences among young men of color.

\section{Limitations}

A few limitations to the current study warrant acknowledgment. First, although our procedures captured the strongest and most prevalent themes, topics that were not probed in greater depth during the interviews and perspectives voiced by a minority of participants were not included in this article. To limit concern about the dependability and credibility of qualitative research methods, our data analysis strategy involved a systematic process for reducing the data to focus on the dominant themes and subthemes in our data. Similarly, because of the qualitative nature of these data, the findings cannot be generalized to other groups of young Black men. Second, we partnered with a well-functioning student mentoring group sponsored by the multicultural student center to develop and implement the YBMen Facebook intervention. Given that many intervention group participants were in- volved in this student group prior to their participation in the YBMen project, they may have felt more inclined to fully participate in the YBMen project. Therefore, our findings may not be particularly reflective of how young Black men who were not already a part of a social group would respond. Third, our team built meaningful relationships with the full-time staff of the multicultural center that organized and managed the Black male student group from which our sample was drawn. These staff members were also influential in disseminating program information and assisting with participant recruitment. The relationships between our study team and the multicultural center staff could have influenced the ways in which the young Black men participated in the YBMen project. Fourth, it is worthy to note that we provided modest cash incentives to all YBMen participants at pretest, posttest, and at the 8-week follow-up, which may have influenced their engagement and completion of the study.

Finally, our association with the multicultural student center staff, the incentives, or our interactions with the participants during data collection and the intervention may have made participants more comfortable with describing things they liked about the intervention, rather than things they may have perceived less favorably. This may have contributed to the greater number of subthemes identified under the "likes" theme than under the "dislikes" and "suggestions" themes. The high participation and retention rates, engagement of participants in the Facebook intervention, and satisfaction about the YBMen intervention expressed in the postintervention interviews and in-person interactions with members of the research team, however, suggest that participants generally found enough aspects of the program to be appealing to remain involved; participate again, if given the option; and recommend participating to their friends. Barring these limitations, our study makes valuable contributions to the potential for using social media platforms to deliver mental health education and social support to young men of color.

\section{Conclusion}

The YBMen project has implications for addressing the social determinants that influence the lives of young Black men (e.g., socioeconomic position, criminal justice system, racial identities, masculine identities) through the use of social media (i.e., Facebook). Identifying and monitoring the influence of these social determinants on mental health and social support can impact future research and practice with young Black men. Similarly, there is value to thinking about how researchers and practitioners can direct resources (such a low-cost, high impact Internet-based interventions) to young Black men appropriately. We believe that many of the challenges young Black men face can be addressed using resources in their own communities and with which they already have familiarity, such as social media tools and popular culture references. The feedback we received from the YBMen Facebook intervention group members will assist us with improving future iterations of the YBMen project and will help to expand current science pertaining to effective intervention strategies for young Black men that are both timely and culturally relevant.

Keywords: Black men; Facebook; intervention; masculinity; mental health 


\section{References}

Adams, H., \& Turner, C. (2013). Inside/outside: A model for social support and rehabilitation of young Black men, Trotter Review, 21(1), Article 3. Retrieved from http://scholarworks.umb.edu/trotter_review/vol21/iss1/3

Belgrave, F. Z., \& Allison, K. W. (2010). African American Psychology from Africa to American. Thousand Oaks, CA: SAGE.

Braveman, P., Egerter, S., \& Williams, D. R. (2011). The social determinants of health: Coming of age. Annual Review of Public Health, 32, 381-398. http://dx.doi.org/10.1146/annurev-publhealth-031210-101218

Chae, D. H., Lincoln, K. D., \& Jackson, J. S. (2011). Discrimination, attribution, and racial group identification: Implications for psychological distress among Black Americans in the National Survey of American Life (2001-2003). American Journal of Orthopsychiatry, 81, $498-$ 506. http://dx.doi.org/10.1111/j.1939-0025.2011.01122.x

Chang, T., \& Yeh, C. J. (2003). Using online groups to provide support to Asian American men: Racial, cultural, gender, and treatment issues. Professional Psychology: Research and Practice, 34, 634-643. http:// dx.doi.org/10.1037/0735-7028.34.6.634

Ellison, N., Vitak, J., Gray, R., \& Lampe, C. (2014). Cultivating social resources on social network sites: Facebook relationship maintenance behaviors and their role in social capital processes. Journal of Computer-Mediated Communication, 19, 855-870.

Englar-Carlson, M., \& Kiselica, M. S. (2013). Affirming the strengths in men: A positive masculinity approach to assisting male clients. Journal of Counseling and Development, 91, 399-409. http://dx.doi.org/10.1002/ j.1556-6676.2013.00111.x

Fernald, D. H., \& Duclos, C. W. (2005). Enhance your team-based qualitative research. Annals of Family Medicine, 3, 360-364. http://dx.doi .org/10.1370/afm.290

Gitlin, L. N., Harris, L. F., McCoy, M. C., Hess, E., \& Hauck, W. W. (2015). Delivery characteristics, acceptability, and depression outcomes of a home-based depression intervention for older African Americans: The get busy get better program. The Gerontologist, 56, 956-965. http://dx.doi.org/10.1093/geront/gnv117

Griffith, D. M., Allen, J. O., Johnson-Lawrence, V., \& Langford, A. (2014). Men on the move: A pilot program to increase physical activity among African American men. Health Education \& Behavior, 41, 164-172. http://dx.doi.org/10.1177/1090198113496788

Griffith, D. M., Gilbert, K. L., Bruce, M. A., \& Thorpe, R. J. (2016). Masculinity in men's health: Barrier or portal to healthcare? In J. J. Heidelbaugh (Ed.), Men's health in primary care (pp. 19-32). New York, NY: Humana Press. http://dx.doi.org/10.1007/978-3-319-260914_2

Griffith, D. M., Gunter, K., \& Allen, J. O. (2011). Male gender role strain as a barrier to African American men's physical activity. Health Education \& Behavior, 38, 482-491. http://dx.doi.org/10.1177/109019 8110383660

Griffith, D. M., Gunter, K. E., \& Watkins, D. C. (2012). Measuring masculinity in research on men of color: Findings and future directions. American Journal of Public Health, 102, S187-S194.

Griffith, D. M., Metzl, J. M., \& Gunter, K. (2011). Considering intersections of race and gender in interventions that address US men's health disparities. Public Health, 125, 417-423. http://dx.doi.org/10.1016/j .puhe.2011.04.014

Grinnell, R. M., Jr., \& Unrau, Y. A. (Eds.). (2014). Social work research and evaluation (10th ed.). New York, NY: Oxford University Press.

Harper, S. R. (2013). Am I my brother's teacher? Black undergraduates, racial socialization, and peer pedagogies in predominantly White postsecondary contexts. Review of Research in Education, 37, 183-211. http://dx.doi.org/10.3102/0091732X12471300

Holt, L. F. (2013). Writing the wrong: Can counter-stereotypes offset negative media messages about African Americans? Journalism \& Mass Communication Quarterly, 90, 108-125. http://dx.doi.org/10.1177/ 1077699012468699
Jackson, B. A. (2012). Bonds of brotherhood: Emotional and social support among college Black men. The Annals of the American Academy of Political and Social Science, 62, 61-71. http://dx.doi.org/10.1177/ 0002716212438204

Jackson, J. S., Knight, K. M., \& Rafferty, J. A. (2010). Race and unhealthy behaviors: Chronic stress, the HPA axis, and physical and mental health disparities over the life course. American Journal of Public Health, 100, 933-939. http://dx.doi.org/10.2105/AJPH.2008.143446

Joe, S., Baser, R. E., Breeden, G., Neighbors, H. W., \& Jackson, J. S. (2006). Prevalence of and risk factors for lifetime suicide attempts among Blacks in the United States. Journal of the American Medical Association, 296, 2112-2123. http://dx.doi.org/10.1001/jama.296.17 .2112

LaCoursiere, S. P. (2001). A theory of online social support. Advances in Nursing Science, 24, 60-77. http://dx.doi.org/10.1097/00012272200109000-00008

Lincoln, K. D., Taylor, R. J., Watkins, D. C., \& Chatters, L. M. (2011). Correlates of psychological distress and major depressive disorder among African American men. Research on Social Work Practice, 21, 278-288. http://dx.doi.org/10.1177/1049731510386122

Mezuk, B., Lohman, M., Dumenci, L., \& Lapane, K. L. (2013). Are depression and frailty overlapping syndromes in mid- and late-life? A latent variable analysis. The American Journal of Geriatric Psychiatry, 21, 560-569. http://dx.doi.org/10.1016/j.jagp.2012.12.019

Mulveen, R., \& Hepworth, J. (2006). An interpretative phenomenological analysis of participation in a pro-anorexia internet site and its relationship with disordered eating. Journal of Health Psychology, 11, 283-296. http://dx.doi.org/10.1177/1359105306061187

Odedina, F., Oluwayemisi, A. O., Pressey, S., Gaddy, S., Egensteiner, E., Ojewale, E. O., . . . Martin, C. M. (2014). Development and assessment of an evidence-based prostate cancer intervention programme for Black men: The W. O. R. D. on prostate cancer video. Ecancermedicalscience, $8,460$.

Oh, H. J., Ozkaya, E., \& LaRose, R. (2014). How does online social networking enhance life satisfaction? The relationships among online support interaction, affect, perceived social support, sense of community, and life satisfaction. Computers in Human Behavior, 30, 69-78. http://dx.doi.org/10.1016/j.chb.2013.07.053

Oliver, W. (2006). "The streets": An alternative Black male socialization institution. Journal of Black Studies, 36, 918-937. http://dx.doi.org/10 $.1177 / 0021934704273445$

Parham, T. A., White, J. L., \& Ajamu, A. (1999). The psychology of Blacks: An African-centered perspective. Upper Saddle River, NJ: Prentice Hall.

Paxton, K. C., Robinson, W. L., Shah, S., \& Schoeny, M. E. (2004). Psychological distress for African American adolescent males: Exposure to community violence and social support as factors. Child Psychiatry and Human Development, 34, 281-295. http://dx.doi.org/10.1023/B: CHUD.0000020680.67029.4f

Payne, J. S. (2012). Influence of race and symptom expression on clinicians' depressive disorder identification in African American men. Journal of the Society for Social Work and Research, 3, 162-177. http://dx .doi.org/10.5243/jsswr.2012.11

Payne, Y. A. (2011). Site of resilience: A reconceptualization of resiliency and resilience in street life-oriented Black men. The Journal of Black Psychology, 37, 426-451. http://dx.doi.org/10.1177/0095798410 394178

Pinnock, C. B., Jones, C., \& the Education Committee of the Australian Prostate Cancer Collaboration. (2003). Meeting the information needs of Australian men with prostate cancer by way of the internet. Urology, 61, 1198-1203. http://dx.doi.org/10.1016/S0090-4295(03)00016-5

Regehr, C., Glancy, D., \& Pitts, A. (2013). Interventions to reduce stress in university students: A review and meta-analysis. Journal of Affective Disorders, 148, 1-11. http://dx.doi.org/10.1016/j.jad.2012.11.026 
Rowling, L. (2006). Adolescence and emerging adulthood (12-17 years and 18-24 years). In M. Cattan \& S. Tilford (Eds.), Mental health promotion: A lifespan approach. New York, NY: McGraw-Hill Education.

Scott, H. M., Pollack, L., Rebchook, G. M., Huebner, D. M., Peterson, J., \& Kegeles, S. M. (2014). Peer social support is associated with recent HIV testing among young Black men who have sex with men. AIDS and Behavior, 18, 913-920. http://dx.doi.org/10.1007/s10461-013-0608-8

Sellers, S. L., Bonham, V., Neighbors, H. W., \& Amell, J. W. (2009). Effects of racial discrimination and health behaviors on mental and physical health of middle-class African American men. Health Education \& Behavior, 36, 31-44. http://dx.doi.org/10.1177/1090198106 293526

Thorpe, R. J., Griffith, D. M., Gilbert, K. L., Elder, K., \& Bruce, M. A. (2016). Men's health in 2010s: What is the global challenge? In J. J. Heidelbaugh (Ed.), Men's health in primary care (pp. 1-18). New York, NY: Humana Press. http://dx.doi.org/10.1007/978-3-319-26091-4_1

Verney, K. (2013). African Americans and U.S. popular culture. New York, NY: Routledge.

Wang, H. H., Wu, S. Z., \& Liu, Y. Y. (2003). Association between social support and health outcomes: A meta-analysis. The Kaohsiung Journal of Medical Sciences, 19, 345-351. http://dx.doi.org/10.1016/S1607$551 \mathrm{X}(09) 70436-\mathrm{X}$

Watkins, D. C. (2012). Depression over the adult life course for African American men: Toward a framework for research and practice. American Journal of Men's Health, 6, 194-210. http://dx.doi.org/10.1177/ 1557988311424072

Watkins, D. C., Allen, J. O., Goodwill, J. R., Noel, B., Goins, T., \& Wilkes, C. (2015, June). The YBMen project at Jackson College. (Unpublished internal document, University of Michigan). Retrieved from https:// gendhrlab.files.wordpress.com/2015/11/ybmen_report_from_jackson final_final.pdf
Watkins, D. C., \& Gioia, D. (2015). Mixed methods research. Pocket Guides to Social Work Research Methods Series. New York, NY: Oxford University Press.

Watkins, D. C., \& Griffith, D. M. (2013). Practical solutions to address men's health disparities. International Journal of Men's Health, 12, 187-194. http://dx.doi.org/10.3149/jmh.1203.187

Watkins, D. C., Hudson, D. L., Caldwell, C. H., Siefert, K., \& Jackson, J. S. (2011). Discrimination, mastery, and depressive symptoms among African American men. Research on Social Work Practice, 21, 269 277. http://dx.doi.org/10.1177/1049731510385470

Watkins, D. C., \& Jefferson, S. O. (2013). Recommendations for the use of online social support for African American men. Psychological Services, 10, 323-332. http://dx.doi.org/10.1037/a0027904

Watkins, D. C., \& Neighbors, H. W. (2007). An initial exploration of what "mental health" means to young Black men. Journal of Men's Health \& Gender, 4, 271-282. http://dx.doi.org/10.1016/j.jmhg.2007.06 .006

Watkins, D. C., Wharton, T., Mitchell, J. A., Matusko, N., \& Kales, H. (2015). Perceptions and receptivity of non-spousal family support: A mixed methods study of psychological distress among older, churchgoing African American men. Journal of Mixed Methods Research. Advance online publication. http://dx.doi.org/10.1177/15586898 15622707

Wester, S. R., Vogel, D. L., Wei, M., \& McLain, R. (2006). African American men, gender role conflict, and psychological distress: The role of racial identity. Journal of Counseling and Development, 84, 419429. http://dx.doi.org/10.1002/j.1556-6678.2006.tb00426.x

Woodward, A. T., Taylor, R. J., \& Chatters, L. M. (2011). Use of professional and informal support by Black men with mental disorders. Research on Social Work Practice, 21, 328-336. http://dx.doi.org/10.1177/ 1049731510388668 